\title{
Could using the zona pellucida bound sperm for intracytoplasmic sperm injection (ICSI) enhance the outcome of ICSI?
}

\author{
De-Yi Liu ${ }^{1,2}$ \\ Asian Journal of Andrology (2011) 13, 197-198; doi:10.1038/aja.2010.179; published online 17 January 2011
}

I $\mathrm{n}$ the recent literature, several interesting articles have been published using the zona pellucdia (ZP)-bound sperm for intracytoplasmic sperm injection (ICSI) to enhance embryo quality, implantation and clinical pregnancy rates. ${ }^{1-3}$ The three studies were performed independently in different ART clinics. In the test group, patients' sibling immature (either germinal vesicle (GV) or metaphase I) oocytes were used to harvest the ZP-bound sperm, which were used for injection of mature oocytes. In the control group, ICSI patients with similar female age and sperm profiles had ICSI with standard sperm selection by the embryologist subjectively choosing the sperm. ${ }^{2,3}$ The results showed that the proportion of high-quality embryos (grades 1 and 2) and implantation rates were higher with ICSI using ZP-bound sperm than with conventional sperm selection. More pregnancies were achieved in the ZP-bound sperm group although clinical (fetal heart detected) pregnancy rates did not reach statistical significance between the two groups, likely due to small sample sizes. These preliminary reports suggest that ZPselected sperm appear to be superior to subjectively selected sperm for ICSI. If this finding can be further verified by a large clinical study, the use of the ZP-bound sperm for ICSI could be applied more widely in ICSI to enhance pregnancy outcome.

In the literature, some studies have been reported that in vitro fertilization (IVF) embryos produced relatively higher implantation and clinical pregnancy rates compared to ICSI embryos. ${ }^{4,5}$ It is possible that some ICSI patients with low implantation or pregnancy

${ }^{1}$ Melbourne IVF, East Melbourne, Vic. 3002, Australia and 2 Department of Obstetrics and Gynaecology University of Melbourne, Parkville, Vic. 3052, Australia Correspondence: Dr D Y Liu (deyi.liu@mivf.com.au) may result from injection of inadequate or poor-quality sperm, for example, with abnormal morphological or DNA damaged sperm. ${ }^{6-9}$ As the majority of ICSI patients are male factor infertility and they have variable abnormal ejaculated sperm, particularly those with high proportion of DNA damaged sperm, subjective manual method is inconsistent or unable to select a most competent sperm for ICSI. Thus it is necessary to develop an objective and efficient method for selection of the most competent sperm for ICSI.

Over the many years since ICSI was first introduced in 1992, ICSI has been using the same sperm preparation methods as conventional IVF, such as swim-up or discontinuous density gradients centrifugation. These classic methods are predominately designed to harvest large numbers of motile sperm and to eliminate non-sperm cells and debris. However, these sperm preparation methods are less or not effective in the selection of sperm with normal morphology and normal DNA. $^{10-13}$ For conventional IVF, it is adequate to inseminate oocytes using motile sperm selected by these methods since the sperm which fertilizes will be further selected through the biological process of spermoocyte interaction, especially sperm-ZP binding. ${ }^{14-17}$ In contrast with conventional ICSI, this additional selection does not occur. The embryologists subjectively select a sperm based only on motility and gross morphology and it is very difficult to select a most competent sperm for injection of an oocyte, in particular for samples with high proportion of DNA damaged sperm. The manual selection of sperm for ICSI is very subjective and inconsistent within and between embryologists.

Although many other different sperm preparation methods have been developed to improve sperm preparation for ICSI, for example, hyaluronic acid (HA) binding and high magnification of microscope combined with computer enlarged image for selection of morphologically normal sperm, ${ }^{9,18,19}$ clinical application of these new methods have not been well evaluated and it is unknown if they are better than the conventional method. It is reported that sperm bound to HA have better morphology and less DNA damage, but using the HA bound sperm for ICSI does not significantly improve implantation and pregnancy. ${ }^{19}$ As the ability of sperm binding to the HA is mainly based on enzyme activity of hyaluronidase which is on the surface of all motile sperm, the majority $(>85 \%)$ of motile sperm are capable of binding to the $\mathrm{HA}^{20}$

In human ejaculate from fertile men, although an average ejaculate contains 100200 million motile sperm, only about $14 \%$ motile sperm are capable of binding to the human $\mathrm{ZP}$ in vitro. ${ }^{16}$ Therefore, there is a very small proportion of motile sperm in human ejaculate that has the capacity of binding to the ZP. Clearly, only those sperm bound to the $\mathrm{ZP}$ have a chance to penetrate the $\mathrm{ZP}$ and then fertilize the oocyte either in vivo or IVF conditions. Thus the majority of motile sperm in ejaculate have no real fertilizing ability in vivo or IVF condition. Although the ability of sperm binding to the $\mathrm{ZP}$ is not necessary and likely only a viable sperm with normal DNA is needed for fertilization with ICSI, many previous studies showed clearly that the human ZP selectively binds sperm with normal morphology. ${ }^{14-17}$ The majority of sperm (average $>92 \%$ ) bound to the ZP have normal nuclear chromatin DNA. ${ }^{17}$ These results suggest that the human ZP possesses the capacity to select normal functioning sperm and therefore, the ZP-bound 
sperm should have a superior quality compared to the general population of motile sperm in medium or scientist subjectively selected motile sperm during conventional ICSI. Thus the use of the ZP-bound sperm, a best proportion of sperm selected biologically through sperm-ZP interaction for ICSI, may produce higher embryo quality, implantation and clinical pregnancy rate than conventional ICSI, which uses a sperm subjectively selected by a scientist.

In clinical ICSI, most patients with controlled ovarian hyperstimulation have $\geqslant 1$ immature oocytes that are not used for ICSI as the immature oocytes cannot produce normal fertilization. ${ }^{2,3}$ The ZP of immature oocyte has similar biological activity as the $\mathrm{ZP}$ of mature oocyte to bind sperm with normal morphology and normal DNA. ${ }^{15,17}$ Therefore, the ZP-bound sperm can be prepared using patients' sibling immature oocytes. In patients without immature oocytes collected, it may be worthwhile using one mature oocyte (if $>6$ oocytes collected) to harvest the ZP-bound sperm. Sperm-ZP binding procedure is very simple and most embryologists can perform it. However, there is limitation of using the ZP-bound sperm for ICSI. For example, some patients may have no sperm bound to the ZP as most ICSI patients have poor ejaculated sperm. In these cases, conventional ICSI using scientistselected sperm for injection of oocytes will be applied and there is no disadvantage to those patients. Also sperm obtained from testicular biopsy or epididymal aspiration will not be suitable for preparation of the ZPbound sperm as sperm from such samples are usually unable to bind to the ZP.

In summary, the ZP-bound sperm appears to be superior or more competent than the general population of motile sperm. Using patients' sibling immature oocytes to harvest ZP-bound sperm for injection of mature oocytes may be a useful approach to improve the selection of sperm for ICSI to enhance ICSI outcomes, particularly those with previous poor outcomes of conventional ICSI or those diagnosed with severe abnormal morphology or DNA damaged sperm. However, controlled clinical trials in larger numbers of subjects are needed to confirm the benefit before using ZP-bound sperm for all ICSI patients routinely.

1 Paes Almeida Ferreira de Braga D, laconelli A Jr, Cássia Sávio de Figueira R, Madaschi C, SemiãoFrancisco $\mathrm{L}$ et al. Outcome of ICSI using zona pellucida-bound spermatozoa and conventionally selected spermatozoa. Reprod Biomed Online 2009; 19: 802-7.

2 Black M, Liu DY, Bourne H, Baker HW. Comparison of outcomes of conventional intracytoplasmic sperm injection (ICSI) and ICSI using sperm bound to the zona pellucida of immature oocytes. Fertil Steril 2010; 93: 672-4.

3 Liu F, Qiu Y, Zou Y, Deng ZH, Liu DY. Use of zona pellucida-bound sperm for intracytoplasmic sperm injection produces higher embryo quality and implantation than conventional intracytoplasmic sperm injection. Fertil Steril; e-pub ahead of print 23 October 2010; doi: 10.1016/j.fertnstert. 2010.09.015.

4 Yoeli R, Ashkenazi J, Orvieto R, Levy T, Ben-Rafael Z et al. Pregnancy potential of embryos from in vitro fertilization compared to intracytoplasmic sperm injection. Gynecol Endocrinol 2000; 14: 253-7.

5 Bhattacharya S, Hamilton MP, Shaaban M, Khalaf Y, Seddler $\mathrm{M}$ et al. Conventional in-vitro fertilisation versus intracytoplasmic sperm injection for the treatment of non-male-factor infertility: a randomised controlled trial. Lancet 2001; 357: 2075-9.

6 Borini A, Tarozzi N, Bizzaro D, Bonu MA, Fava L et al. Sperm DNA fragmentation: paternal effect on early post-implantation embryo development in ART. Hum Reprod 2006; 21: 2876-8.

7 Lopes S, Sun JG, Jurisicova A, Meriano J, Casper RF. Sperm deoxyribonucleic acid fragmentation is increased in poor-quality semen samples and correlates with failed fertilization in intracytoplasmic sperm injection. Fertil Steril 1998; 69: 528-32.

8 Speyer BE, Pizzey AR, Ranieri M, Joshi R, Delhanty JD et al. Fall in implantation rates following ICSI with sperm with high DNA fragmentation. Hum Reprod 2010; 25: 1609-18.

9 Berkovitz A, Eltes F, Yaari S, Katz N, Barr I et al. The morphological normalcy of the sperm nucleus and pregnancy rate of intracytoplasmic injection with morphologically selected sperm. Hum Reprod 2005; 20: 185-90.

10 Liu DY, Baker HW. Tests of human sperm function and fertilization in vitro. Fertil Steril 1992; 58: 465-83.

11 Angelopoulos T, Moshel YA, Lu L, Macanas E, Grifo JA et al. Simultaneous assessment of sperm chromatin condensation and morphology before and after separation procedures: effect on the clinical outcome after in vitro fertilization. Fertil Steril 1998; 69: 740-7.

12 Sakkas D, Manicardi GC, Tomlinson M, Mandrioli M, Bizzaro $D$ et al. The use of two density gradient centrifugation techniques and the swim-up method to separate spermatozoa with chromatin and nuclear DNA anomalies. Hum Reprod 2000; 15: 1112-6.

13 Tomlinson MJ, Moffatt O, Manicardi GC, Bizzaro D, Afnan $\mathrm{M}$ et al. Interrelationships between seminal parameters and sperm nuclear DNA damage before and after density gradient centrifugation: implications for assisted conception. Hum Reprod 2001; 16: 2160-5.

14 Liu DY, Baker HW. Morphology of sperm bound to the zona pellucida of human oocytes that failed to fertilize in vitro. J Fertil Reprod 1992; 94: 71-84.

15 Liu DY, Garrett C, Baker HW. Clinical application of sperm-oocyte interaction tests in in vitro fertilizationembryo transfer and intracytoplasmic sperm injection programs. Fertil Steril 2004; 82: 1251-63.

16 Liu DY, Garrett C, Baker HW. Low proportions of sperm can bind to the zona pellucida of human oocytes. Hum Reprod 2003; 8: 2382-9.

17 Liu DY, Baker HW. Human sperm bound to the zona pellucida have normal nuclear chromatin assessed by Acridine Orange fluorescence. Hum Reprod 2007; 22: 1597-602.

18 Bartoov B, Berkovitz A, Eltes F, Kogosowski A, Menezo $Y$ et al. Real-time fine morphology of motile human sperm cells is associated with IVF-ICSI outcome. J Androl 2002; 23: 1-8.

19 Nasr-Esfahani MH, Razavi S, Vahdati AA, Fathi F Tavalaee M. Evaluation of sperm injection procedure based on hyaluronic acid binding ability on ICSI outcome. J Assist Reprod Genet 2008; 25: 197-203.

$20 \mathrm{Ye} \mathrm{H}$, Huang GN, Gao Y, Liu DY. Relationship between sperm-hyaluronan binding assay and fertilization rate in conventional in vitro fertilization. Hum Reprod 2006; 21: 1545-50. 